\title{
Mapping Controls on a 2D User Drawn Racetracks Driving Game - An Usability Assessment
}

\section{Fernando Rocha}

Department of Informatics

Engineering, University of

Coimbra, Portugal

fmrocha@student.dei.uc.pt

Pedro Machado Santa

Department of Informatics

Engineering, University of

Coimbra, Portugal

pgms@student.dei.uc.pt

Jorge C.S. Cardoso

CISUC, Department of

Informatics Engineering,

University of Coimbra,

Portugal

jorgecardoso@dei.uc.pt

\section{Luís Lucas Pereira}

Department of Informatics

Engineering, University of

Coimbra, Portugal

Ipereira@dei.uc.pt

\section{Licínio Roque}

Department of Informatics

Engineering, University of

Coimbra, Portugal

lir@dei.uc.pt

Permission to make digital or hard copies of part or all of this work for personal or classroom use is granted without fee provided that copies are not made or distributed for profit or commercial advantage and that copies bear this notice and the full citation on the first page. Copyrights for third-party components of this work must be honored. For all other uses, contact the Owner/Author.

CHI PLAY EA '19, October 22-25, 2019, Barcelona, Spain.

(c) 2019 Copyright is held by the owner/author(s).

ACM ISBN 978-1-4503-6871-1/19/10.

DOI: https://doi.org/10.1145/3341215.3356302

\begin{abstract}
In this paper, we present the results from an

experiment designed to evaluate the user experience of five different control schemes (buttons, steering wheel, joystick, joystick+button and accelerometer-based tilting) in a 2D top-down racing mobile game designed specifically for children. The experiment was conducted with 24 children from ages 6 to 14, both male and female, and with different levels of experience with mobile devices and mobile gaming. We present the results from our observations from the participants' interaction with the game as well as the results from a questionnaire and interview. Results indicate that the joystick controller provides a better experience than the proposed alternatives to inexperienced players.
\end{abstract}

\section{Author Keywords}

Children; game design; mobile games; playtesting.

\section{CSS Concepts}

- Human-centered computing Usability testing;

\section{Introduction}

Gaming is an enormous part of our daily lives, either by playing a game ourselves or even by watching someone else play. From casual games, to games designed for 
players to spend hundreds of hours, there is a kind of videogame for everyone, making them playable by children and older people alike. Technology gives children the possibility to express ideas that otherwise they wouldn't have the capabilities to [0]. Creative thinking and imagination are at the core of game design so children should be empowered to create interesting videogames. But videogame development requires technical skills, such as computer programming which can pose a learning challenge.

We are currently developing Playsketch [15], a mobile tool to provide children with the opportunity to design their own games based on common functional blocks and a common skill - drawing on paper. Our first videogame style is a 2D top-down racing game, where players draw racetracks on paper and then play the game using their own drawing as a basis for track design. An initial playtesting session of the current version of the game revealed problems involving the controls available to the players. For instance, some players tried to tilt the devices to make the car turn while others didn't have the coordination to use two buttons to turn the car. As a result, we have implemented new car controls into the game and evaluated them. In this paper, we describe the game along with its new control options, the methodology and experiment conducted with children, and discuss its results.

The contributions of this paper are the comparison of five controls for a $2 \mathrm{~d}$ top-down mobile game for children and an assessment of the subjective user preference of said control schemes. Since Playsketch works with user drawn racetracks, and the player perspective rarely aligns with the car orientation, driving controls often become a mapping challenge.

\section{Related Work}

\section{Controls}

One of the most important characteristics that define a game's experience is the way the player interacts with it or "the instrument of expression for the player into the game world" [22]. Game designers understand the importance of good control design, how those controls are mapped into actions in-game, and how they impact the player experience [20] including different aspects of it such as overall enjoyment, perceived personality [2] and sense of immersion [3]. All these studies support the idea that choosing the right controller for a game can have a great impact on the experience that its designer is trying to convey.

Seven games from Apple's App Store (Micro Racing [8], Doodle Kart [10], Vs. Racing 2 [11], Mini Turbo GP

[19]) and Google's Play Store (Head to Head Racing [4], High Octane [7], Retro Racing [14]) were analysed in order to understand what types of controllers are used in the top-down racing games genre for mobile devices. These games were chosen via an online search on the above referenced mobile app stores, the only requirement was being free to play.

Six of these games had the option to turn the car using left and right virtual buttons on the touch screen. Of these six, three had the option to turn the car using an accelerometer-based tilting control. Tilting the device left or right would turn the car left or right. The game that did not have virtual buttons as an option used a virtual joystick to steer and accelerate the car. Five games used automatic acceleration as the primary 


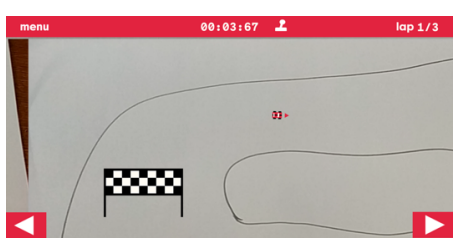

Figure 1: The virtual buttons controller option to give speed to the car, even though three of those had the option to turn it off. Turning automatic acceleration off would add another virtual button to accelerate the car.

\section{Usability Evaluation with Children}

Playtesting is one of the most used testing methods in game companies for usability evaluation [16, 17]. Playtesting involves gathering a large group of players with the aim to play a game, giving the evaluators useful feedback to improve the overall experience of said game. This feedback can be objective, through evaluation of metrics, such as task performance times or number of errors, or subjective, with the usage of questionnaires and interviews to understand players' interpretation and emotional response. Playtesting should accompany the game development process as soon as possible, to avoid bad core game design [6]. Later, playtesting may serve as a final mark to consider a game as ready to launch. Playtesting has proven to be very effective and cost-efficient for detecting issues even for low-budget independent developers [13]

Still, designing games for children can be difficult because a game aimed at 9-year-olds might create barriers for a 6-year-old. Conducting usability tests with children can be difficult, as many children cannot express their feelings towards the game being evaluated [5]. On the other hand, methods like constructive interaction have proven to be more effective than think-aloud to ascertain usability problems [1]. Taking the time for children to gain confidence with the researcher through activities outside the user test also helps them become more open to sharing what they think is faulty with the system [24]. Studies have shown that children can identify usability problems even with low-fidelity prototypes and that developers can have confidence that the results will transfer to higher fidelity [21].

\section{The Playsketch 2D Racing Game Style}

The game we are studying is a work-in-progress 2D top-down racing game for mobile devices. The main intention of the game is to invite children to use their imagination and create their own "levels" by drawing racetracks on paper. The game has two play phases: the creation phase and the racing phase.

In the creation phase, the player draws a racetrack in a piece of paper. Obstacles in the middle of the racetrack or decoration outside it can be drawn freely. After the player draws her level to preference, she takes a picture of the drawing using the mobile device. A game algorithm processes the drawing and creates a level.

Using the game interface, the player adds a finish line element. At this stage, the player can also add game elements such as acceleration tracks - to provide a speed boost - and oil spills - to make the car spin uncontrollably - to the racetrack. After the level is designed, a 3-lap racing begins.

One big problem about finding the right controller for this game play scenario is its changeable level design. Most games have certain control schemes fit to their level design, or even the level design is thought out in order to "map naturally to" the player controls. In the current version, the player controls a car by using two virtual buttons on the screen which turn it right and left, as shown in Figure 1. Acceleration of the car is fully automatic. In previous playtests, it was detected that this control scheme was not easily adopted by younger children as they would either press the buttons 


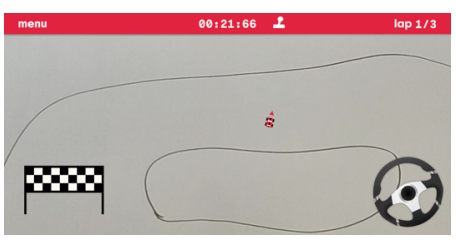

Figure 2: The steering wheel controller

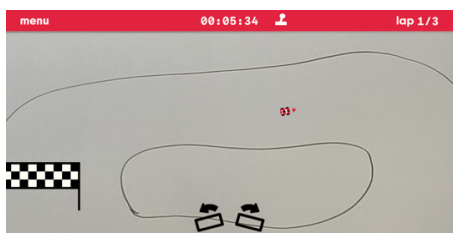

Figure 3: The accelerometer-

based tilting controller

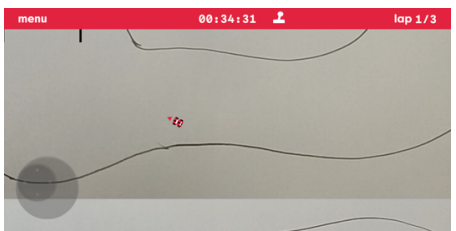

Figure 4: The analog joystick controller

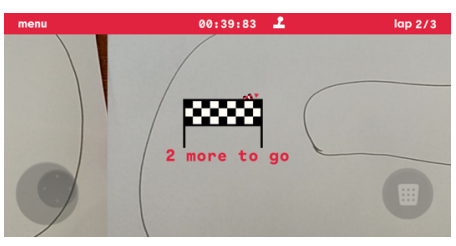

Figure 5: The joystick+button controller randomly, press the buttons as if they were directional, i.e. right button should move the car to the right of the screen, or keep one button always pressed and press and release the other.

We found it was necessary to study alternative control schemes: steering wheel, accelerometer-based tilting, joystick with an acceleration button and joystick without the acceleration button. These alternatives were based on research of similar games $[4,7,8,10$ $11,14,19]$.

In the steering wheel controller, depicted in Figure 2, acceleration is fully automatic and turning the wheel clockwise turns the car right, and vice-versa. In the accelerometer-based tilting controller (Figure 3), acceleration is also automatic and tilting the device left or right turns the car left or right. The joystick controllers are the only ones without automatic acceleration. In the joystick without acceleration button case, Figure 4, the acceleration is calculated through the distance between the thumbstick and the centre of the joystick, following an analog-like behaviour, while with the button, Figure 5, the acceleration is mapped through that virtual button. The direction of the car is directly mapped from the angle of the thumbstick in both cases. A small moving arrow rotates around the car to show direction in context.

\section{Experiment Design}

The main goal of this experiment is to understand which of the controllers is the best fit for the race design scenario specific to this game. Additionally, we want to understand player overall experience while interacting with the game.
Evaluation Procedure

The experiment followed a within-subjects study design regarding the five different controllers: virtual buttons

(current controller), steering wheel, joystick, joystick+button, and tilting.

Participants were children, aged 6 to 14 , contacted with 3 weeks notice and provided with an informed consent form, to be signed by their respective tutor, so the sessions could be both audio and video recorded. The video recordings consisted of face cams recorded from the device used in the experiment. Sessions occurred in a school, a place to learn but also play, borrowing class time, as it was not possible to schedule otherwise. Each session involved two simultaneous participants, as constructive interaction with acquainted dyads provides better results with children than the think-aloud method [1]. Of 55 children contacted only 24 were able to take part, due to time constraints. During the session day, students were randomly selected by their teachers to leave the classroom in pairs to meet with the evaluator in an adjacent room. At the beginning of each session, we explained the purpose and the system. Then, participants were asked about their age, gender, experience with mobile devices and mobile gaming.

Participants would then play the same level of the game to complete three laps with each controller, randomly selected by the evaluator. The device used was a Xiaomi Mi A2 with a face recording app. The sound came from the built-in speakers turned up around $50 \%$ volume. The evaluator gave no information on how to use each controller and the participants were not given any time to train with each controller. The evaluator would take notes about 
players' comments, posture and difficulties. After the first two sessions, it was necessary to cut the time with the interaction with each controller to 50 seconds instead of the three laps completion objective, as players would take too long to finish the objective with some controls, becoming stressful for some players.

After the playing phase, the participants answered a questionnaire about their experience playing the game. The questionnaire used a 3-point Likert scale, with each point corresponding to the responses "Yes", "More or less" (or "Maybe") and "No". As children have difficulty providing answers when the Likert response formats are based on numbers [12], and tend to choose only the extreme options $[9,18]$, this approach was chosen over the 5-point number based Likert scale. It was also necessary for the evaluator to ask the questions and fill the participants' answers in order to shorten the duration of this step, as the participants would take a long time reading the questions, even though they could answer them almost immediately.

At the end of each session, participants were engaged in a semi-structured interviews, starting by ordering the controllers by their preference, using the Fun Sorter and the Again-Again Table from the Fun Toolkit [18]. Again-Again Table was discarded after 4 sessions since participants only chose their preferred controller.

\section{Participants}

The 24 participants, identified in Table 1, were familiar with mobile devices (smartphones and tablets) and most owned a device with, exception of four. Seven participants used mobile devices every day, 2 more than twice a week, 11 once or twice a week, and 4 used them less than once a week. Twenty stated that gaming is their main activity and watching gaming videos online is usual. Eight participants referred usually playing racing games, mostly with videogame consoles or personal computers.

\section{Results and Discussion}

Through observations, the following problems were detected with each controller:

- Virtual buttons: as the buttons are set at the lower part of the screen, they usually go undetected for a while, making the players try to tilt the device; random pressing of the buttons; continuous pressing of one button without lifting the finger (causing the car to spin).

- Steering Wheel: this control gives the players better control over fine movements, but children like to see the steering wheel rotate so they usually overshoot their curves.

- Joystick: no major problems detected with this controller; some participants referred it was too small.

- Joystick with acceleration button: inexperienced players tend to accelerate - stop - turn - stop accelerate, instead of accelerating and turning at the same time.

- Tilting: some participants tried to press the visual aid that indicated to tilt the device; fine movements are hard to achieve using this control, tilting too little barely turns the car while tilting too much makes the car spin. It is extremely difficult for children to find the "sweet spot" were the tilt is just right to control the car.

The questionnaires were handed after both participants finished playing using all the available controllers: 


\begin{tabular}{|c|c|c|}
\hline Ages & Number & Gender \\
\hline [6-7] & 10 & $\begin{array}{r}4 \text { Male \& } 6 \\
\text { Female }\end{array}$ \\
\hline [8-9] & 10 & $\begin{array}{r}4 \text { Male \& } 6 \\
\text { Female }\end{array}$ \\
\hline 11 & 4 & $\begin{array}{r}2 \text { Male } \& 1 \\
\text { Female }\end{array}$ \\
\hline 14 & 1 & 1 Male \\
\hline
\end{tabular}

Table 1: Age and gender

distribution of the participants

\begin{tabular}{|c|c|c|c|c|c|}
\hline $\begin{array}{l}0 \\
0 \\
0 \\
0 \\
\frac{1}{0} \\
\frac{0}{0} \\
\frac{1}{2}\end{array}$ & 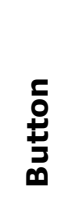 & $\begin{array}{l}\bar{d} \\
\stackrel{ \pm}{3}\end{array}$ & $\begin{array}{l}\frac{y}{u} \\
\frac{n}{n} \\
0 \\
0\end{array}$ & $\begin{array}{l}m \\
+ \\
\mathbf{n}\end{array}$ & $\stackrel{ \pm}{F}$ \\
\hline $\begin{array}{l}\text { First } \\
\text { (Most } \\
\text { Fun) }\end{array}$ & 1 & 5 & 9 & 8 & 1 \\
\hline Second & 3 & 1 & 7 & 11 & 2 \\
\hline Third & 7 & 6 & 4 & 3 & 4 \\
\hline Fourth & 7 & 9 & 4 & 1 & 3 \\
\hline $\begin{array}{l}\text { Fifth } \\
\text { (Least } \\
\text { Fun) }\end{array}$ & 6 & 3 & 0 & 1 & 14 \\
\hline
\end{tabular}

Table 2: Frequency of controller preference
- Six participants stated what it was more or less fun to play our game, every other participant stated that it was fun. One participant said that he got bored and 4 others more or less.

- Most of the players who regularly play racing games said that the car moved too slowly while less experienced players stated the opposite. Five participants expressed that the car did not move too slowly either too fast.

- Half of the participants mentioned the game was moderately or too hard.

- Eight participants expressed their disinterest in playing the game a second time.

At the end of each session, participants were asked to order the used controllers by their preference. The results are shown in Table 2 . The results indicate a clear preference for both joystick options while most voted the accelerometer-based tilting as the worst controller.

Due to the closeness between the preference of both joystick options, the factor of playing experience was considered. Out of the 8 participants that regularly play racing games, 5 selected joystick+button as their preferred controller while none chose the joystick. This means that inexperienced players prefer the joystick without the acceleration button over the other options. These results in conjunction with the evaluator observations of the participants' performance, show that the joystick provides better results as some participants could not perform both turning and accelerate actions at the same time.

Most participants stated that they had no specific reason to prefer a certain controller while some stated the following reasons:
- Virtual buttons: one participant stated ease of use.

- Steering wheel: one participant stated that it gives the sensation of driving a real car.

- Joystick: ease of use and only one hand is necessary.

- Joystick with acceleration button: the button gives the impression of greater speed.

- Tilt: one participant said that turning the device from side to side is fun.

One flaw with this experiment is that, while the order of the controllers was random between participants, the usage of each one was not fully balanced. This had no major consequences on the results because the joystick and tilt controllers were the most used ( 6 times) in last place and obtain completely different results.

\section{Conclusions}

We have presented the results from an experiment designed to compare five control schemes typically implemented in top-down racing mobile games.

Results indicate that the joystick options provide more comfort and are preferred by both inexperienced and experienced players alike, even though experienced players prefer better control over the acceleration of the car via a complementary button.

These results can be used by game developers who wish to develop top-down racing mobile games for children.

As an additional note, this study should be interpreted with care, as the evaluation was performed without regarding the balance of usage of controllers and without further tuning of the parameters of each control that performed poorly. 


\section{References}

1. Benedikte S. Als, Janne J. Jensen, and Mikael B. Skov. 2005. Comparison of think-aloud and constructive interaction in usability testing with children. In Proceedings of the 2005 conference on Interaction design and children (IDC '05), 9-16. http://doi.acm.org/10.1145/1109540.1109542

2. Max Birk and Regan L. Mandryk. 2013. Control your game-self: effects of controller type on enjoyment, motivation, and personality in game. In Proceedings of the SIGCHI Conference on Human Factors in Computer Systems (CHI '13). 685-694. http://doi.acm.org/10.1145/2470654.2470752

3. Paul Cairns, Jing Li, Wendy Wang, and A. Imran Nordin. 2014. The influence of controllers on immersion in mobile games. In Proceedings of the SIGCHI Conference on Human Factors in Computer Systems (CHI '14), 371-380. http://doi.acm.org/10.1145/2556288.2557345

4. Craigs games. 2013. Head to Head Racing. Game [Android].

5. Afke Donker and Pieter Reitsma. 2004. Usability testing with young children. In Proceedings of the 2004 conference on Interaction design and children: building a community (IDC '04), 43-48. http://doi.acm.org/10.1145/1017833.1017839

6. Tracy Fullerton. 2014. Game design workshop: a playcentric approach to creating innovative games. AK Peters/CRC Press.

7. Fry-up Productions. 2017. High Octane. Game [Android].

8. Geemzo. 2012. Micro Racing. Game [iOS].

9. Gareth Griffiths. 2014. Child's Play: Playtesting with children in the world of Skylanders. Video. Retrieved November 20, 2018 from https://www.gdcvault.com/play/1020348/Child-sPlay-Playtesting-with

10. J2sighte. 2010. Doodle Kart. Game [iOS].
11. MdLabs AB. 2015. Vs. Racing 2. Game [iOS].

12. David Mellor and Kathleen A. Moore. 2013. The use of Likert scales with children. Journal of pediatric psychology 39, 3 (April 2014), 369-379. https://doi.org/10.1093/jpepsy/jst079

13. Perjman Mirza-Babaei, Naeem Moosajee, and Brandon Drenikow. 2016. Playtesting for indie studios. In Proceedings of the $20^{\text {th }}$ International Academic Mindtrek Conference (AcademicMindtrek '16), 366-374. https://doi.acm.org/10.1145/2994310.2994364

14. Mr Qwak Limited. 2015. Retro Racing. Game [Android]

15. Luís Lucas Pereira, Pedro Machado Santa, and Licínio Roque. 2013. What you draw is what you play: a natural approach to participatory game creation. Paper submitted and accepted for the $9^{\text {th }}$ Student Interaction Design Research Conference (SIDeR '13). Retrieved September 28, 2018 from http://sider2013.au.dk/fileadmin/sider2013/0126paper.pdf

16. Mikko Rajanen and Joonas Nissinen. 2015. A survey of Game Usability Practices in Northern European Game Companies. Selected Papers of the IRIS. Issue $\mathrm{Nr} 6$ (2015). Paper 8. http://aisel.aisnet.org/iris2015/8

17. Mikko Rajanen and Juho Tapani. 2018. A Survey of Game Usability Practices in North American Game Companies. In Proceedings of the $27^{\text {th }}$ International Conference on Information Systems Development (ISD2018).

18. Janet C. Read and Stuart MacFarlane. 2006. Using the fun toolkit and other survey methods to gather opinions in child computer interaction. In Proceedings of the 2006 conference on Interaction design and children (IDC '06), 81-88. http://doi.acm.org/10.1145/1139073.1139096

19. RedBit Games. 2014. Mini Turbo GP. Game [iOS]. 
20. Jesse Schell. 2008. The Art of Game Design: $A$ Book of Lenses. Morgan Kauffman, Burlington, MA.

21. Gavin Sim, Brendan Cassidy, and Janet C. Read. 2013. Understanding the fidelity effect when evaluating games with children. In Proceedings of the $12^{\text {th }}$ International Conference on Interaction Design and Children (IDC '13), 193-200. http://doi.acm.org/10.1145/2485760.2485769

22. Steve Swink. 2008. Game Feel: A Game Designer's Guide to Virtual Sensation. CRC/Press.
23. Nicola Yelland. 2011. Reconceptualising Play and Learning in the Lives of Young Children. In

Australasian Journal of Early Childhood 36, 2 (June 2011), 4-12.

https://doi.org/10.1177/183693911103600202

24. Yusrita Mohd Yusoff, Monica Landoni, and Ian Ruthven. 2010. Assessing fun: young children as evaluators of interactive systems. Retrieved 16 November, 2018 from

https://pure.strath.ac.uk/ws/portalfiles/portal/5801 341/final_proceedings.pdf 